\title{
Infrasound Signal Analysis of Satellite Launch
}

\author{
PANG Xin-Liang ${ }^{1, a^{*}}$, CHENG Xian-You ${ }^{1, b}$, MA Yan ${ }^{1, c}$, CHEN Hui-Xing ${ }^{1, a}$, LIU \\ $\mathrm{Xin}^{1, \mathrm{a}}$ and $\mathrm{LI} \mathrm{Xin}^{1, \mathrm{a}}$ \\ ${ }^{1}$ State Key Laboratory of NBC Protection for Civilian, Beijing,102205 P.R. China \\ a pangxinliang@sina.com, b13366831492@163.com, cole_my@126.com
}

\begin{abstract}
Keywords: nuclear explosion detection; infrasound measurement; satellite launch; infrasound signal characteristics;.

Abstract. This paper measures the infrasonic signal of a Satellite Launch event and analyzes the signal characteristics. Using four infrasonic sensors to finish the satellite launch event monitoring tests, the experimental results show that difference between the infrasonic signal of satellite launch event and the infrasonic signal of background is very clear, the signal amplitude is dozens times of the background in the range of tens of kilometers; the closer, the frequency distribution of the wider, almost throughout the infrasonic frequency range in the range of several kilometers; obvious frequency concentration by increasing distance, the frequency focus in $7-12 \mathrm{~Hz}$ at 50 kilometers, and as the distance increases, the frequency down .
\end{abstract}

\section{Introduction}

Infrasound is a frequency lower than $20 \mathrm{~Hz}$ sound waves, some natural phenomena produce infrasound signal ${ }^{[1][2]}$, such as volcanoes, earthquakes, landslides, mudslides, lightning, etc. ${ }^{[3][4]}$, human activities will produce infrasound signal also, such as nuclear explosions, mining blasting, chemical explosion, the satellite launch, the aircraft carrier runs, supersonic aircraft flight, these natural or anthropogenic activities have a very strong shock waves, for example: nuclear explosion shock wave, the difference between the pressure shock wave compression zone and normal atmospheric pressure is called "overpressure" or "positive pressure", the difference between the pressure shock sparse area and normal atmospheric pressure is called "negative". In the process of shock wave propagation in the atmosphere, overpressure shock wave front surface gradually decreases the velocity of the wavefront is gradually reduced, the overpressure duration gradually increased, also will be gradually widened waveform; and negative pressure gradient slow and is a perturbation, it is acoustic properties. With the continued increase in the spread of distance shock wave continues to stretch wider, when the speed is reduced to the speed of sound wavefront size, then the shock wave transforms into sound waves. Explosion sound waves is generally regarded as a spherical wave in the near field, and can be regarded as plane wave when it spreads to distant far field, it is aperiodic, continuous spectrum. Certain energy nuclear explosion sound waves propagate in the atmosphere in the process of gradually with increasing distance attenuation by atmospheric absorption, reflection, refraction, dispersion, frequency dispersion; sound intensity reduces by transmission to a certain distance, wider wavelength, lower frequency, gradually degenerate into a infrasonic waves (frequency less than $20 \mathrm{~Hz}$ ), atmospheric nuclear explosion infrasound signal forming.

Infrasound detection technology to monitor nuclear explosions event is an effective information technology to get explosion basic parameters and it is very practical approach. In 1994, the UN Conference on Disarmament in Geneva, Comprehensive Nuclear-Test-Ban Treaty, infrasound monitoring technologies was one of four monitoring technologies(infrasound, hydroacoustic, seismic, radionuclide) in comprehensive ban on nuclear tests.

In this paper, satellite launch infrasonic signal characteristic is analyzed as a disturbance signal to nuclear explosion infrasonic signal. 


\section{Infrasound Monitoring Equipment and Layout}

Satellite launch event in this paper is a low orbit satellite from the Satellite Launch Center in 2014, the satellite launch tower latitude and longitude: longitude $102^{\circ} 1^{\prime} 40$ ", latitude $28^{\circ} 14^{\prime} 45^{\prime \prime}$.

The experiment uses an infrasonic sensor ${ }^{[5][6]}$ (sensor No. 7) and one infrasound monitoring array (triangle infrasound array: sensors No. 1, No. 2 and No. 4), Total 4 infrasonic sensors monitor the satellite launch event. Sensor NO.7 is located in latitude and longitude: longitude:102 ${ }^{\circ}{ }^{\prime} 52.74 "$, latitude: $28^{\circ} 12^{\prime} 40.68^{\prime \prime}$; triangle of infrasound array located in latitude and longitude of sensor NO.1:

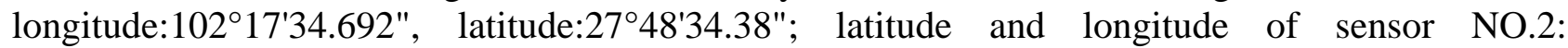

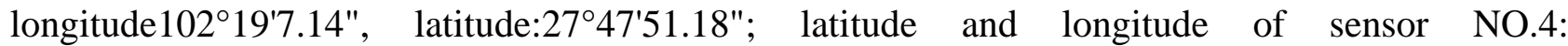

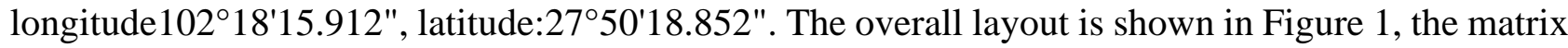
layout is shown in Figure 2:

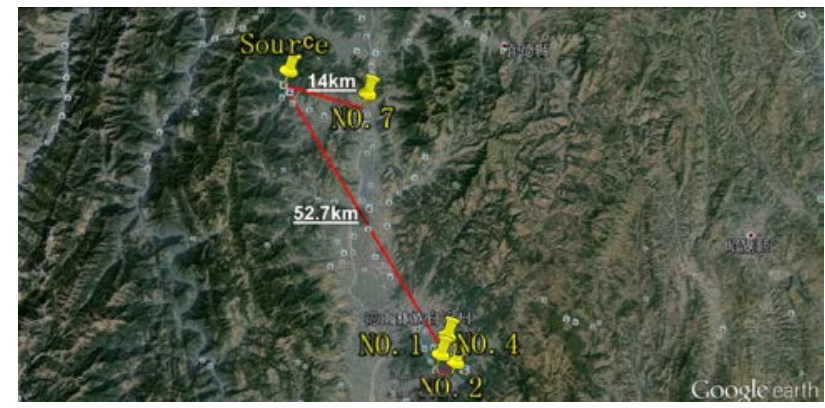

Fig. 1 Satellite launch event monitoring equipment layout.

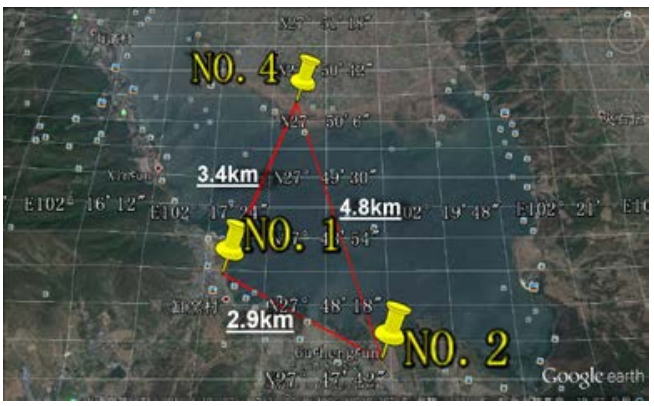

Fig. 2 The triangular matrix layout map.

The distance is 14 kilometers between sensor NO.7 and satellite launch tower, lie a hill, the sensor is set up the foothills; triangular matrix is $52.7 \mathrm{~km}$ far away from the satellite launch tower, triangular celssl space between each other, respectively $2.9 \mathrm{~km}, 3.4 \mathrm{~km}, 4.8 \mathrm{~km}$.

\section{Infrasound monitoring Signal Analysis}

\subsection{The 1st station monitoring signal analysis}

Infrasound signals collected by the 1st station as shown in Figure 3, in Figure 3 the Extreme infrasound intensity appears at October 24, 2014 02:05:33. The original signal background removal and band-pass filtered signal shown in Figure 4, the sound pressure Extreme is 26.482Pa in figure 4. Zooming out figure 4, satellite launch event infrasound signal waveform shown in Figure 5. Infrasound power spectrum of satellite launch event in Figure 5 is shown in Figure 6, the frequency range of the event is focused on $9-11 \mathrm{~Hz}$, frequencies of the two extreme points are $10.077 \mathrm{~Hz}$ and $10.197 \mathrm{~Hz}$.

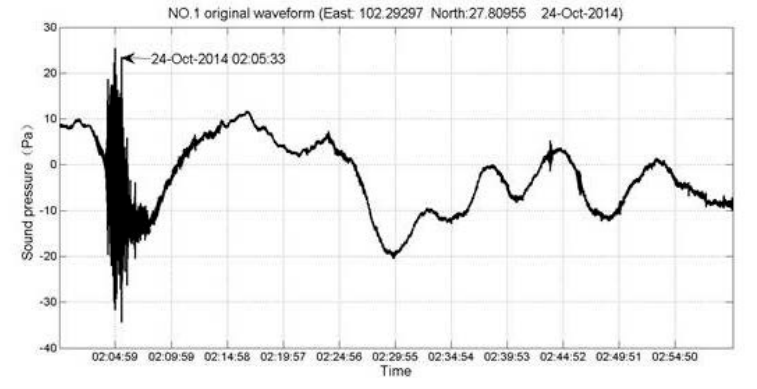

Fig. 3 Original infrasound signal for sensor NO.1.

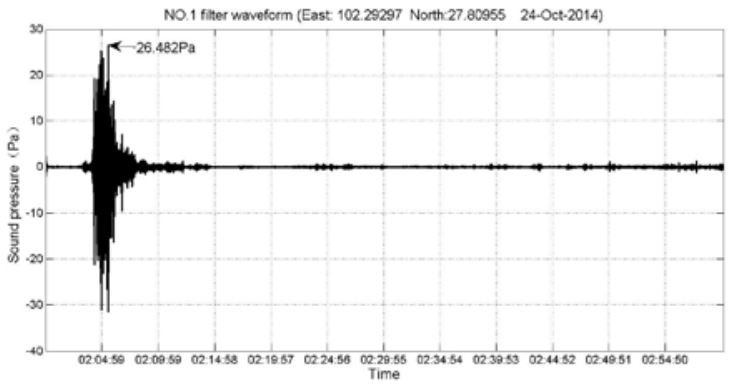

Fig. 4 Filter infrasound signal for sensor NO.1 


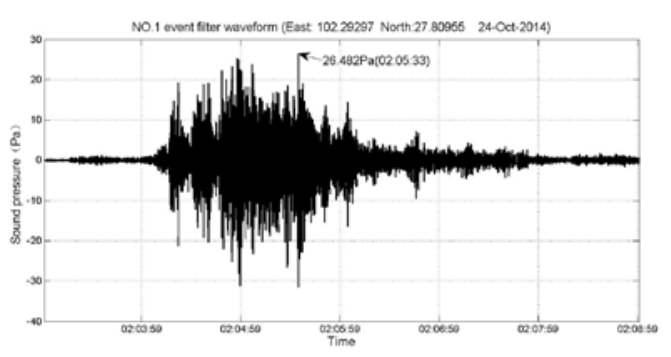

Fig. 5 The satellite launch event filtered signal for sensor NO.1

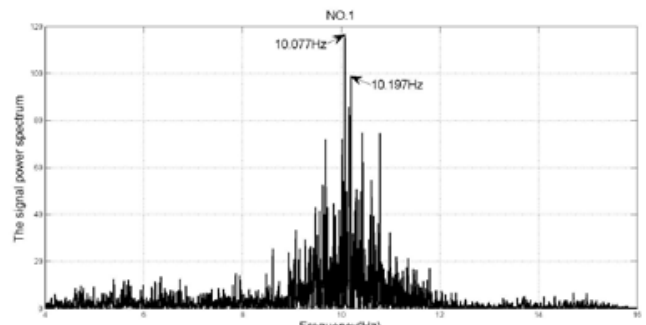

Fig. 6 signal power spectrum of sensor NO.1 for satellite launch event

\subsection{The 2nd station monitoring signal analysis}

Infrasound signals collected by the 2nd station as shown in Figure 7, in Figure 7 the Extreme infrasound intensity appears at October 24, 2014 02:05:20. The original signal background removal and band-pass filtered signal shown in Figure 8, the sound pressure Extreme is 23.7074Pa in figure 8. Zooming out figure 8, satellite launch event infrasound signal waveform shown in Figure 9. Infrasound power spectrum of satellite launch event in Figure 9 is shown in Figure 106, the frequency range of the event is focused on $7-10 \mathrm{~Hz}$, frequencies of the two extreme points are $8.364 \mathrm{~Hz}$ and $8.766 \mathrm{~Hz}$.

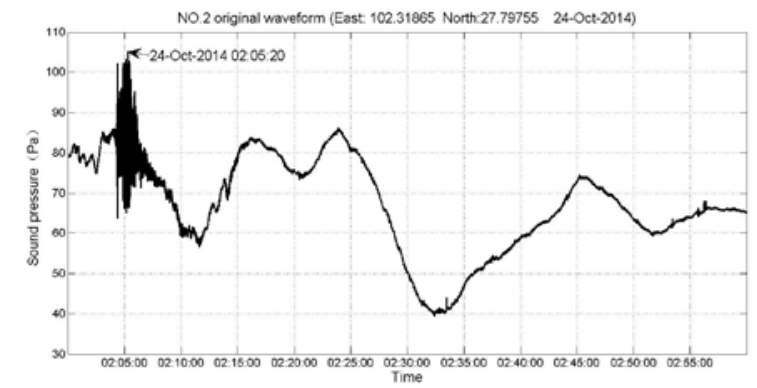

Fig. 7 Original infrasound signal for sensor NO.2

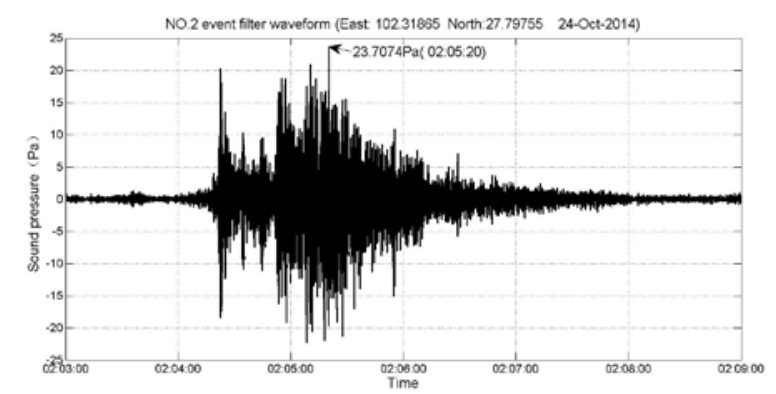

Fig. 9 The satellite launch event filtered signal for sensor No.2

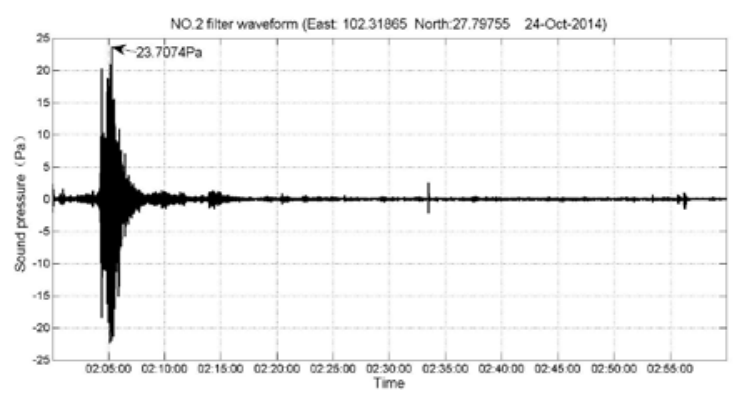

Fig. 8 Filter infrasound signal for sensor NO.2

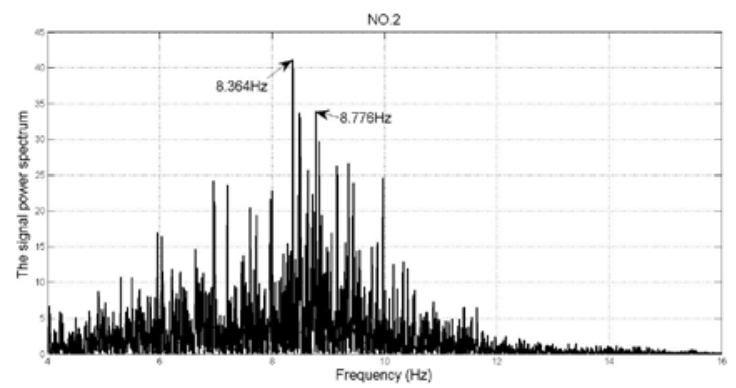

Fig. 10 signal power spectrum of sensor NO.2 for satellite launch event

\subsection{The 3rd station monitoring signal analysis}

Infrasound signals collected by the 1st station as shown in Figure 11, in Figure 11 the Extreme infrasound intensity appears at October 24, 2014 02:04:47. The original signal background removal and band-pass filtered signal shown in Figure 12, the sound pressure Extreme is 40.8959Pa in figure 12. Zooming out figure 12, satellite launch event infrasound signal waveform shown in Figure13. Infrasound power spectrum of satellite launch event in Figure 13 is shown in Figure 14, the frequency range of the event is focused on $10-12 \mathrm{~Hz}$, frequencies of the two extreme points are $11.230 \mathrm{~Hz}$ and $11.719 \mathrm{~Hz}$. 


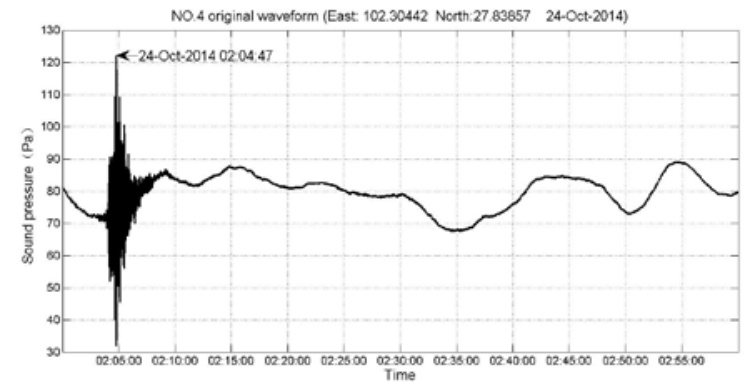

Fig. 11 Original infrasound signal for sensor NO.4

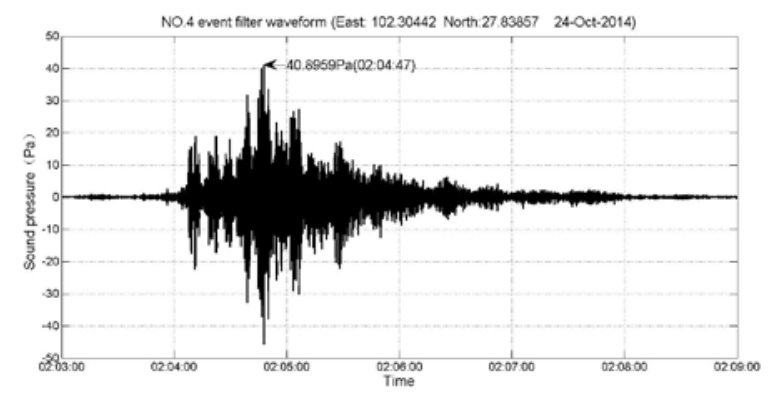

Fig. 13 The satellite launch event filtered signal for sensor No.4

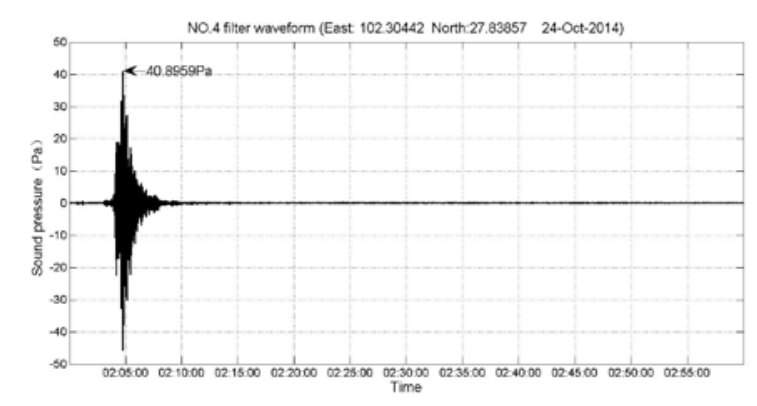

Fig. 12 Filter infrasound signal for sensor NO.4

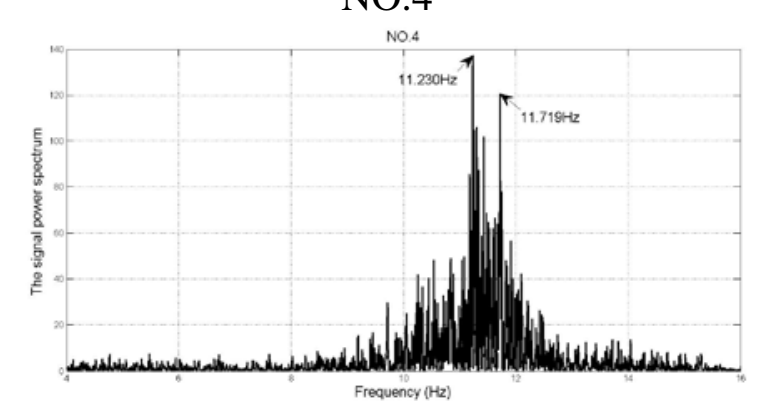

Fig. 14 signal power spectrum of sensor NO.4 for satellite launch event

\subsection{The 4th station monitoring signal analysis}

Infrasound signals collected by the 1st station as shown in Figure 15, in Figure 15 the Extreme infrasound intensity appears at October 24, 2014 02:02:11. The original signal background removal and band-pass filtered signal shown in Figure 16, the sound pressure Extreme is 29.1445Pa in figure 16. Zooming out figure 16, satellite launch event infrasound signal waveform shown in Figure 17. Infrasound power spectrum of satellite launch event in Figure 17 is shown in Figure 18, the frequency range of the event is almost the overall infrasound frequency range, frequencies of the two extreme points are $2.462 \mathrm{~Hz}$ and $11.632 \mathrm{~Hz}$.

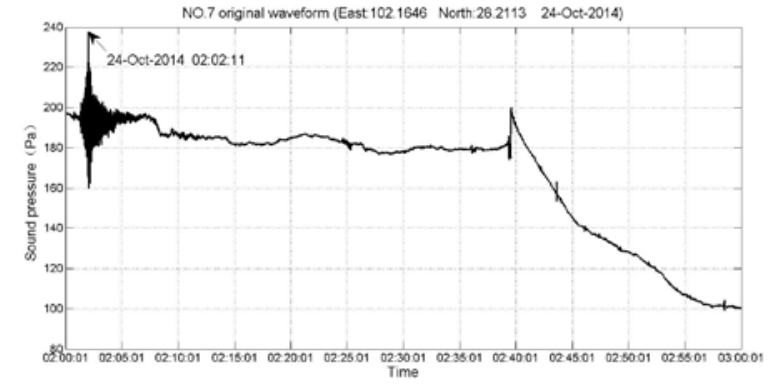

Fig. 15 Original infrasound signal for sensor NO.7

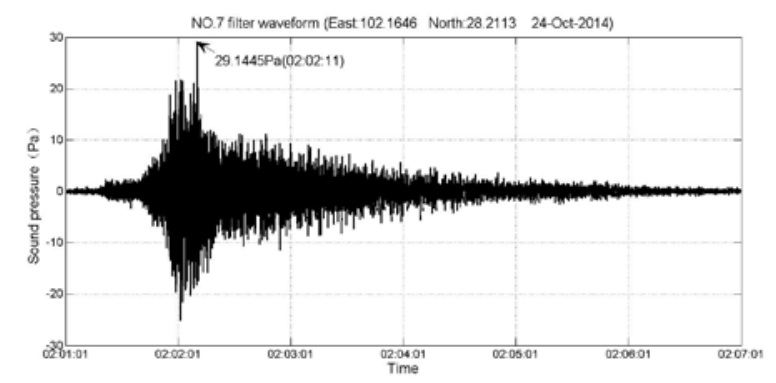

Fig. 17 The satellite launch event filtered signal for sensor No.7

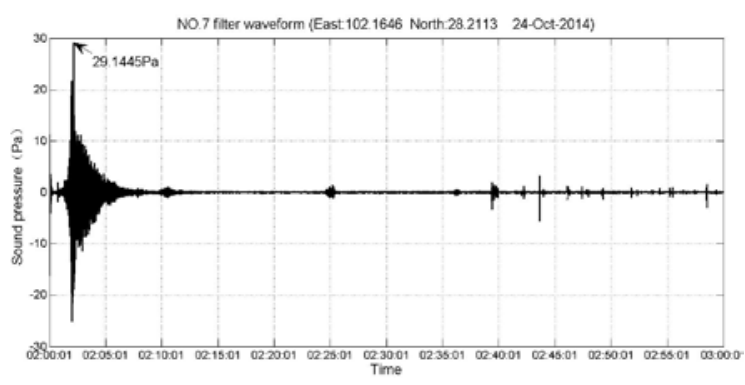

Fig. 16 Filter infrasound signal for sensor

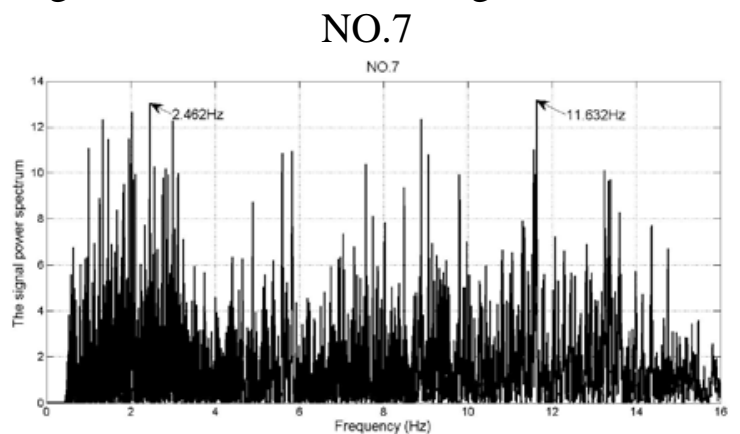

Fig. 18 signal power spectrum of sensor NO.7 for satellite launch event 


\section{CONCLUSION}

Infrasound signal of satellite launch event is very clear, and can be easily distinguished from the background by the signal amplitude in the range of tens of kilometers, the closer, the frequency distribute wider, almost overall infrasound frequency range, obvious frequency concentration by increasing distance, frequency locals mainly in $7-12 \mathrm{~Hz}$ at about $50 \mathrm{~km}$, and as the distance increases, the main frequency down, the main frequency of the sensor NO.2 is lower than that of the sensor NO.1 and NO.4.

\section{References}

[1] Alexis Le Pichon, Elisabeth Blanc, Alain Hauchecorne. Infrasound monitoring for atmospheric studies. Springer Dordrecht Heidelberg London New York.2009.

[2] M.Willis, M.Garces, et al. Infrasonic observations of open ocean swells in the Pacific: Deciphering the song of the sea, Geophysical Research Letters, VOL.31,L19303,doi:10.1029/2004GL020684, 2004.

[3] Xinliang Pang, Ying Wang, et al. Technology research of infrasonic signal acquisition and pretreatment for identification of nuclear explosion. ICONE21, 2013.

[4] Xinliang Pang, Ying Wang, et al. Infrasound measurement and signal character analysis of chemical explosion. AMTIE2014, 2014.

[5] David J.Brown, Rodney, et al. Automatic infrasonic signal detection using the Hough transform. Journal of Geophysical Research, VOL.113,D17105, doi:10.1029/2008JD009822,2008.

[6] D.Ponceau, P.Dupont, S.Peyraud. Recent developments in absolute infrasound Sensors. 2007 Infrasound technology workshop, Novemberl3-16, 2007, Tokyo, Japan. 